\title{
Effects of Pregnancy on Uptake and Distribution
}

1. MAC for volatiles is decreased up to $40 \%$ in pregnancy

(a) Rate of induction is faster due to greater minute ventilation and reduced functional residual capacity (FRC)

2. Pregnant women have a larger volume of distribution due to expanded extracellular volume and total body water, so recovery from many anesthetic drugs is faster

3. The potency of some medications is increased due to decreased levels of albumin and alpha 1-acid glycoprotein for drug binding leading to more unbound drug concentration in plasma 